\title{
EXPANSION-DEFORMATION JOINTS CALCULATION SYSTEMS FOR BRIDGES WITH SIMPLY SUPPORTED SUPERSTRUCTURES, ADMISSIBLE TOLERANCES.
}

\section{Mihai Netid}

Master Degree

Roads, Materials, and Mechanization in Construction

Road design engineer

Abstract. Climate and natural factors act on joint cover devices; means of transportation, through contact with motor vehicles tires on deformation joints; exploitation factors (conditions and level of maintenance of the bridge constructions); the displacements of the superstructure heads at intermediate and marginal bents.

Keywords: displacements, superstructure, joint cover device, temperature.

\section{TYPES OF ACTIONS AND EFFORTS ON DEFORMATION JOINTS.}

For most bridge constructions, after approximately one year and a half of bridge exploitation, visible degradation of joint coverage devices could be seen. Following visual analysis, in most cases the degradation process begins with the formation of cracks between the joint cover device and the road clothing. Afterwards, due to the increased dynamic actions on the runway and the deformation joint from the passage of traffic, holes are formed in the road clothing in the transition zone.

The main reason of dynamic actions appearance of high intensity is the difference between the upper surface of the joint cover device and the surface of the road system (,Step”).

Vertical loads. An important factor in the work of bridge construction to the action of vertical loads also largely acts and the type of support devices. In this regard, neglecting the correct choice of the supporting devices can lead to the worsening of the superstructures work. 
For the continuous superstructures, it is necessary to arrange the supports, which will work on the large horizontal displacements.

\section{DETERMINATION OF DISPLACEMENTS IN THE TEMPERATURE} RANGE.

Fundamental for the calculation of the deflection joint movements are the maximum and minimum temperature values of the superstructure construction of the bridge. These temperature values determine the range of change in the dimensions of the deformation joint opening (the temperature change calculation range $\Delta t$ ), which is necessary for the correct and rational choice of the type of joint cover device (and its dimensions). When performing laboratory work in order to simplify as maximum and minimum temperatures, the maximum and minimum air temperature values $\left(T_{\max }\right.$ şi $T_{m i n}$ ), in accordance with the requirements [1].

It is necessary to stipulate, that in reality such simplification almost always can be considered incorrect [2], because the temperature of the superstructure construction, as a rule, is not equal to the ambient air temperature (for example, because of the temperature inertia of the superstructure material, the sunstroke - the action on the runway with the sun's rays, as well as other factors).

The calculation interval of temperature range is counted as the sum of the maximum and minimum air temperature modules $\left(T_{\max }\right.$ and $\left.T_{\min }\right)$ [3]:

$$
\Delta t=\left|T_{\max }\right|+\left|T_{\min }\right|,
$$

The mounting temperature of the deformation joint is determined by direct measurement of the construction of the bridge superstructure at the stage of arrangement of the joint cover device with the following corrections of the size of the deformation joint apertures before fixing it to the superstructure elements.

When choosing and arranging the joint cover device it is necessary to know, first of all, the calculation dimensions of the movements of the superstructure heads which are simply supported and the admissible movement sizes for this type of construction. 
Determination of temperature movements of the simple leaned end of the superstructure:

$$
\Delta_{t}=\gamma_{T} * \alpha * L * \Delta_{t}
$$

Where: $\gamma_{T}-$ safety coefficient for temperature actions;

$\alpha-$ the linear expansion coefficient of temperature, $K^{-1}$;

$L$ - calculation length of the superstructure, where the displacements originate;

$\Delta_{t}-$ the range of change of the calculation temperatures from $T_{\min }$ to $T_{\max }$ considering the increase of this interval due to solar overheating of the structure, including the different distribution and the temperature in the section of the element.

The safety coefficient for temperature actions $\gamma_{T}$ is adopted according to p. 6.32 SP53.13330.2011 [4] as for deformation and temperature action and is equal to 1,2.

Apart from this, for the convenience of the traffic participants the size of the opening of the deformation joint should not exceed $8.0 \mathrm{~cm}$, because in the case of "entering" of the vehicles tires between the metal heads of the deformation joint, the dynamic coefficient increases $(1+\mu)$, which is an additional factor, increasing the actions of the loads on the roadside part of the bridge. This coefficient increases significantly in the case of non-compliance with the flatness of the microrelief due to degradations in the area of the joint cover device.

\section{DETERMINATION OF THE DISPLACEMENTS OF THE SUPERSTRUCTURE HEADS AT DIFFERENT TYPES OF ACTIONS AND EFFORTS}

Classification of actions by the criterion of "movements of the superstructure heads" (or "displacements") involves division in the following types of displacements (figure 3.1) [2, 5]: 

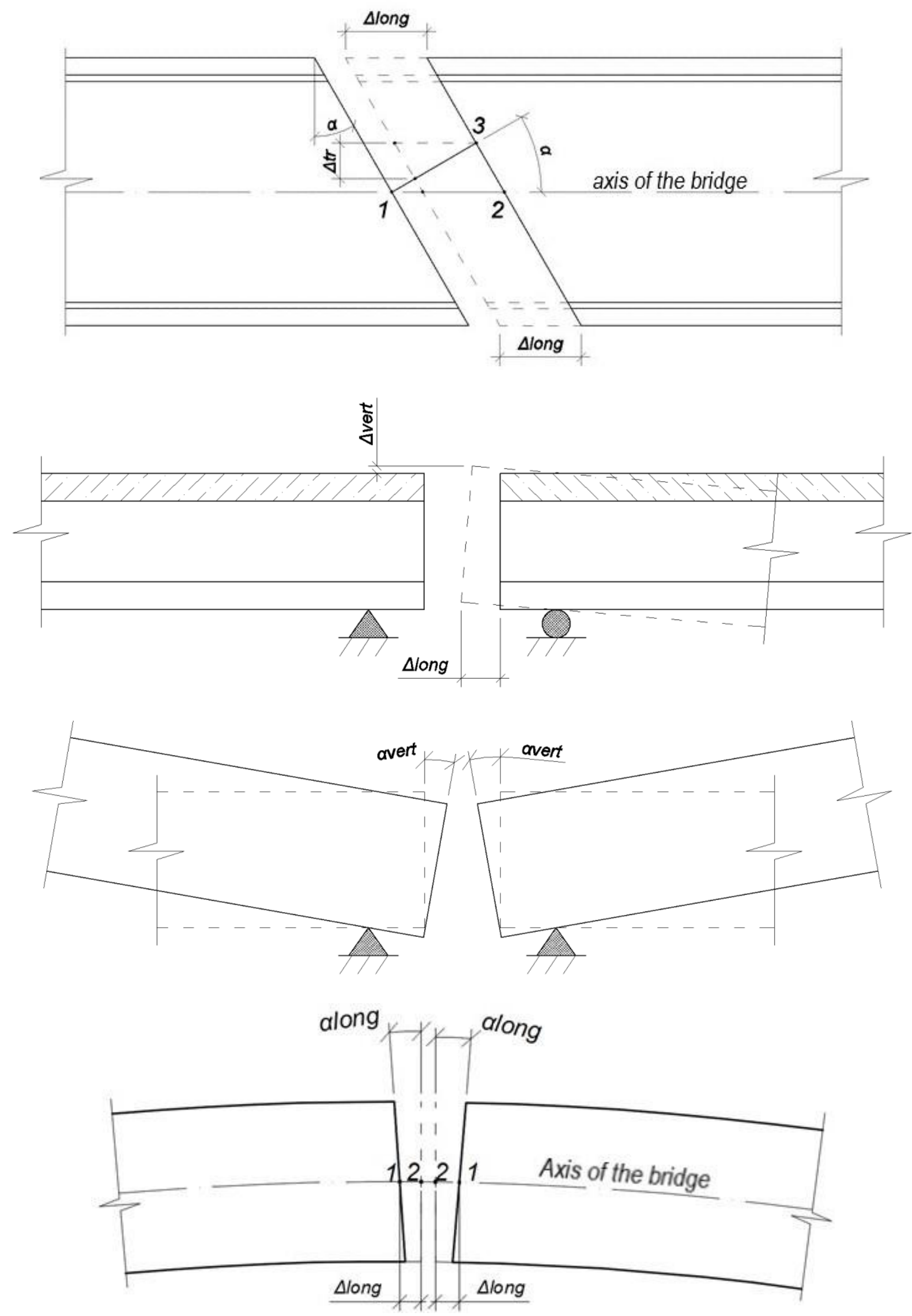

Figure 3.1. Examples of s superstructure heads displacement. 
a) Horizontal, longitudinal and transverse linear displacements of the superstructure ends with equal spacing along the joint $\Delta_{\text {long }}$ și $\Delta_{\text {trans }}$ (figure 3.1.a; plan view of the angular joint) [3].

\section{1) Temperature actions.}

The evaluation of temperature influence on steel and reinforced concrete superstructures can be done with the following formula:

$$
\Delta_{l}=1,2 * \alpha * \Delta_{t} * l
$$

Where: 1,2 - the safety coefficient for the temperature actions [2];

$\alpha-$ coefficient of expansion from the action of temperature, for concrete [6] is equal to: large fraction crushed granite stone, $\alpha_{b . g .}=9,5 * 10^{-6} * K^{-1}$, with large fraction crushed limestone $\alpha_{b . l .}=6,8 * 10^{-6} * K^{-1}$; for steel [6]: $\alpha_{o .}=$ $1 \ldots 1,1 * 10^{-6} * K^{-1}$.

$\Delta_{t}-$ computation range for temperature change for respective area;

$l$ - length of computation ,chain”, where the movements accumulate;

The length of the "chain" computation, from which the action movement of the temperature accumulates, is called the length of the bridge sector, the sector between adjacent fixed supports, with the condition, that the deformation zone is located in the respective area (figure 3.2):

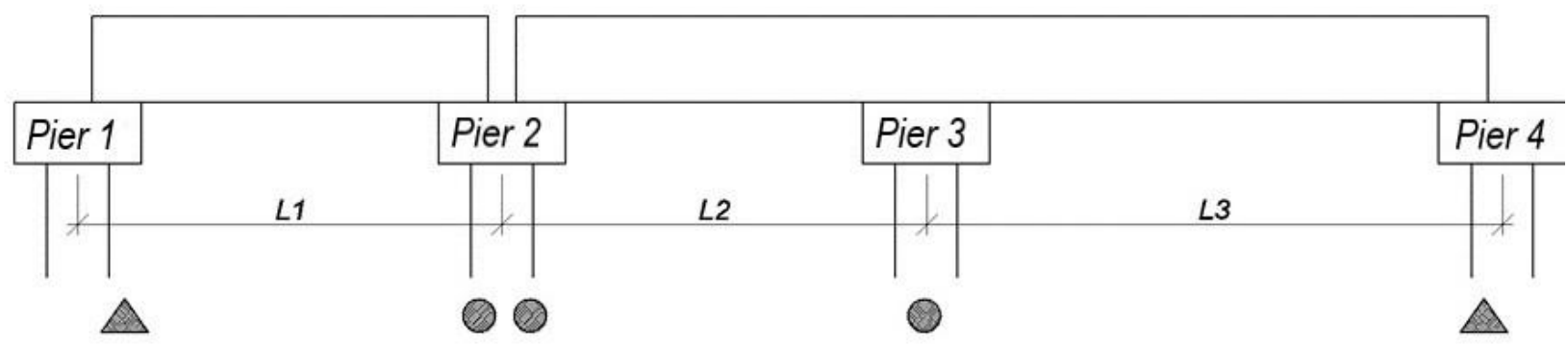

Figure 3.2. Scheme of length calculation for displacements collection

In figure 3.2, the deformation joint is located at pier cap nr.2, fixed supportspiers nr. 1 and 4 . Then the length of the "chain" calculation will be equal with, $l=$ $l_{1}+l_{2}+l_{3}$. 
If the superstructures are of different types (eg mixed superstructures), then the evaluation of their movements will be done separately, using each time 3.1 formula.

In the calculation of mixed steel and reinforced concrete superstructures at temperature actions according p.5.10 from [4], the difference between the temperature of steel elements and reinforced concrete in section must be taken into account. Calculations of temperature actions are allowed to be performed by receiving sectional variations of unchanged temperature values along the superstructure and based on the following maximum values after normative of the temperature differences of the reinforced concrete slab and the steel construction [3]:

a) For superstructures with „I” steel beams with overhead way:

- If the steel temperature values are superior to reinforced concrete, and the beam undergoes heating from solar radiation at $30^{\circ} \mathrm{C}$ and more;

- If the steel temperature values are superior to reinforced concrete only that the beam is not subjected to heating from solar radiation $-15^{\circ} \mathrm{C}$;

- If the steel temperature values are inferior to reinforced concrete: $-15^{\circ} \mathrm{C}$;

b) For lattice girder superstructures and with overhead way:

- If the temperature values of the steel elements of the lattice beams are superior to the reinforced concrete, regardless of the sunlight conditions: $15^{\circ} \mathrm{C}$;

- If the temperature values of the steel elements of the lattice beams are inferior to the reinforced concrete: $-10^{\circ} \mathrm{C}$.

c) For superstructures with main beams that have full heart or lattice beams, and placed between them a reinforced concrete slab and a middle way or lower:

- If the steel temperature values are superior to reinforced concrete: $20^{\circ} \mathrm{C}$;

- If the steel temperature values are inferior to reinforced concrete: $-15^{\circ} \mathrm{C}$.

The mixed section of reinforced concrete and steel can be conventionally divided by part of steel and reinforced concrete. At temperature, these parts tend to lengthen with different values, and it happens because of the different temperature 
values of these different elements and materials from which they were executed. An important factor is the total length change $\Delta_{l}$ (Figure 3.3), calculated with the recording of the particularities signaled.

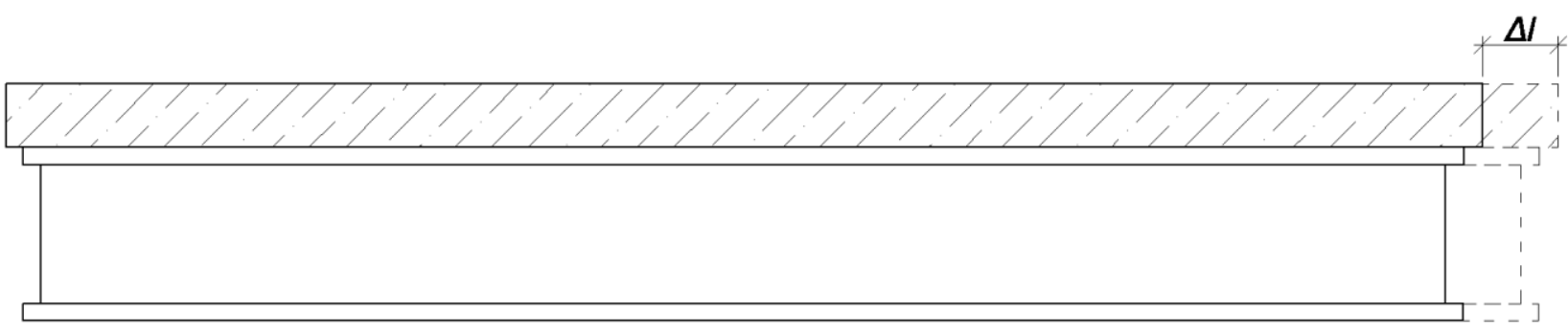

Figure 3.3. Mixed beam of steel and reinforced concrete

So, when changing the temperature values, there is an expansion or compression effort in these elements, depending on which of the elements tends to grow louder or less.

Suppose that the mixed steel beam and reinforced concrete (Figure 3.4) has the temperature $t_{1}$. The modulus of elasticity of the reinforced concrete element $E_{l}$, the area of its section $-F_{l}$, effective length $-l_{l}$, coefficient of thermal expansion - $\alpha_{l}$, initial temperature $-t_{1}$. The characteristic parameters for the steel element are equal to $E_{2}, F_{2}, l_{2}, \alpha_{2}, t_{2}$. At thermal action, the reinforced concrete part of the beam is heated to the temperature - $t_{3}$, and that of steel - to the temperature $t_{4}$.

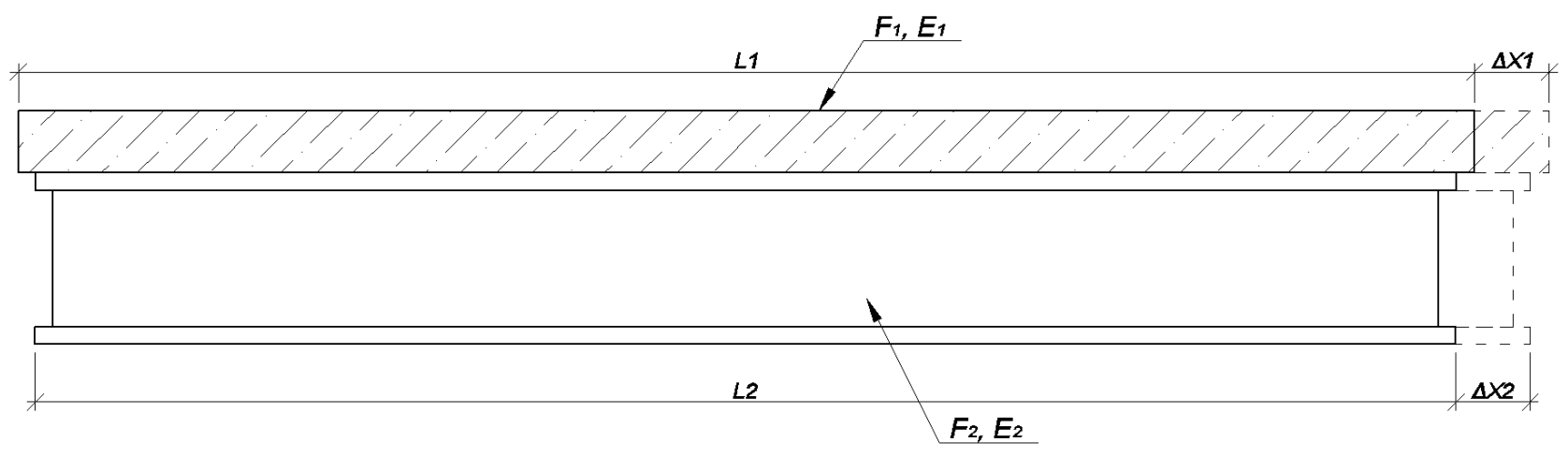

Figure 3.4. Expansion actions of the mixed steel and reinforced concrete beams

In case the mixed steel and reinforced concrete section will be separated, both parts will be expanded with different sizes: reinforced concrete slab - with $\Delta x_{1}=$ $\alpha_{1} *\left(t_{3}-t_{1}\right) * l_{1}$ or $\Delta x_{1}=\alpha_{1} * \Delta T_{1} * l_{1}$, where $\Delta T_{1}=t_{3}-t_{1}$, and the steel beam with $\Delta x_{2}=\alpha_{2} *\left(t_{4}-t_{2}\right) * l_{2}=\alpha_{2} * T_{2} * l_{2}$, where $\Delta T_{2}=t_{4}-t_{2}$. Just that both 
sides of the mixed beams are a whole, therefore the less dilated element prevents dilation of the other (and vice versa), as a result reactive force occurs $R$, which compresses the second element. It will be analyzed the system of the two elements (component parts), which can be presented as the scheme of calculation of the mixed steel and reinforced concrete beams (Figure 3.5). Prolongation required $\Delta l$ will be determined for the reinforced concrete slab (Figure 3.5) according to formula (3.2):

$$
\Delta l=\Delta x_{1}+\Delta l_{R 1},
$$

Where under Hook's law:

$$
\Delta l_{R 1}=\frac{R * l_{1}}{E_{1} * F_{1}}
$$

The steel beam also acts as force $R$ on the plate side (Figure 3.5), (equal to the force $R$ acting on the plate), and considering thermal action on the steel beam and is reflected in equality (3.4) and (3.5):

$$
\begin{gathered}
\Delta l=\Delta x_{2}+\Delta l_{R 2}, \\
\Delta l_{R 2}=\frac{R * l_{2}}{E_{2} * F_{2}},
\end{gathered}
$$
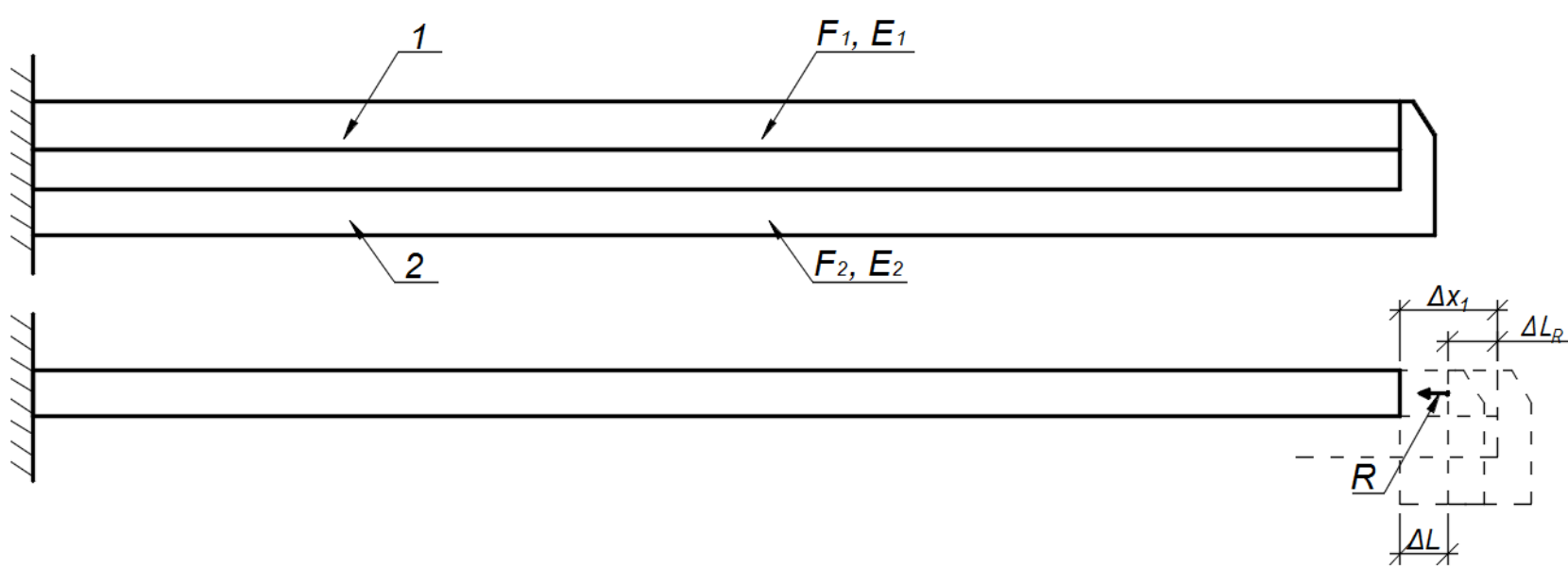

Figure 3.5. Beam calculation and plate movement scheme. 


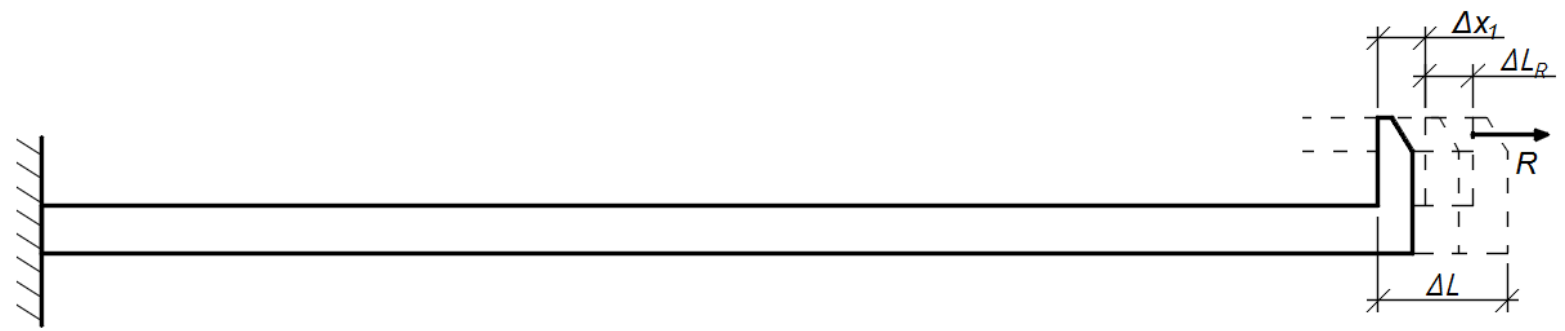

Figure 3.6. Moving the steel beam.

Because the parts to the left of equation (3.2) and (3.4) are equal, equaling them, we find the $R$-value of the deformation compatibility condition [7]:

$$
R=\frac{\Delta x_{1}-\Delta x_{2}}{\frac{l_{1}}{E_{1} * F_{1}}-\frac{l_{2}}{E_{2} * F_{2}}},
$$

Substituting the formula (2.3) în (2.2), we have:

$$
\Delta l=\Delta x_{1}+\frac{R * l_{1}}{E_{1} * F_{1}}
$$

, which transforms with the formula (3.6), is added the safety coefficient for thermal deformations, equal to 1,2 [2] and is founded the displace searched $\Delta l$ (3.8).

$$
\Delta l=1,2 *\left(\Delta x_{1}+\frac{\Delta x_{1}-\Delta x_{2}}{\frac{l_{1}}{E_{1} * F_{1}}-\frac{l_{2}}{E_{2} * F_{2}}} * \frac{l_{1}}{E_{1} * F_{1}}\right),
$$

or:

$$
\Delta l=1,2 *\left(\Delta x_{1}+\frac{l_{1}\left(\Delta x_{1}-\Delta x_{2}\right)}{l_{1}-l_{2} * \frac{E_{1} * F_{1}}{E_{2} * F_{2}}}\right),
$$

where: $\Delta l$ - displacements of the mixed steel and reinforced concrete beam superstructure from the thermal action considering the requirements p. 5.10. [4];

$\Delta x_{1}=\alpha_{1} *\left(t_{3}-t_{1}\right) * l_{1}-$ thermal displacements for reinforced concrete slab;

$\Delta x_{2}=\alpha_{2} *\left(t_{4}-t_{2}\right) * l_{2}-$ thermal displacements for steel beam.

\section{2) Slow flow of concrete.}


When calculating the displacements of the heads of the superstructure it can be assumed that the deformation due to the slow flow of the concrete takes place within 10 years (or more if the beams are additionally loaded temporarily) [2].

It can be considered that the action of the slow flow of concrete on the displacements of the heads of the superstructure will be simplified, the lower limit of the temperature change calculation range by $15^{\circ} \mathrm{C}$ will be decreased [8]

\section{3) Concrete contraction}

If the deformation joints are to be mounted after a certain time of the bridge construction (as is usually the case), it is necessary to calculate the time during which deformations from contraction will occur, which can be adopted conditionally for 5 years for the construction site in temperate and cold climates, and after the expiration of time these deformations can be considered conventionally stopped. However, the rest of the contraction deformations, and hence the magnitude of the displacements, are taken into consideration by considering the age of the concrete at the stage of setting up the deformation joint cover device according to table 3.1 taken from [2]

Tabel 3.1. Deformations from normative contraction, $\%$, depending on the concrete age, months.

\begin{tabular}{|r|c|c|c|c|c|c|c|c|c|}
\hline Climate regions & 1 & 3 & 6 & 12 & 18 & 24 & 36 & 48 & 60 \\
\hline Temperate and cold climates & 30 & 50 & 60 & 70 & 77 & 84 & 92 & 96 & 100 \\
\hline Southern parts & 40 & 55 & 67 & 80 & 90 & 95 & 100 & - & - \\
\hline
\end{tabular}

In the table 3.1 is indicated the percentage of deformations in the concrete contractions from the relative deformations of the concrete contractions $\varepsilon_{s n}$ at the present time.

The contraction of superstructures in reinforced concrete elements causes horizontal displacements, but the contraction of the reinforced concrete slab of mixed steel and reinforced concrete beam - horizontal and vertical displacements [2, 5]. 
Calculation dimensions of the superstructure heads displacements from the concrete contraction and slow flow are determined by multiplying the normative values by the safety coefficient, $\gamma=1,1[2,4]$.

\section{4) Acceleration and braking of transport.}

Horizontal displacements of the deformation joints can be caused by the vertical movements of the infrastructure piles (following the concrete contraction), the efforts made by acceleration and braking of vehicles.

Concerning the efforts of the brakes and the acceleration of the transport, then the displacements from their action can be calculated approximately with Hook's formula [7]:

$$
\Delta_{l o n g}^{a c, f r}=\frac{S^{a c, f r} * l}{E * F}
$$

where: $l-$ the full length of the superstructure;

$E-$ modulus of the superstructure material elasticity;

$F-$ the cross-sectional area of the superstructure.

$S^{a c, f r}$ - braking and traction forces of the transport, adopted according $\mathrm{p}$. $2.19 *[4]$ in the following cases:

- In calculating the deformation joints of road bridges to roads: category I-III and intra-urban bridges - 75,46 kN;

- Category IV and V - 53,9 kN (0,5 K tf).

\section{CONCLUSION}

Following the analysis of the expansion joint calculation systems for bridges with plain bearing superstructures, the need to apply them in the context of designing a bridge construction is noticed. Factors that will influence the choice of type will be natural (maximum and minimum temperatures, seismicity of the design area, soil compaction of the foundation, etc.), of construction (longitudinal profile of the bridge 
construction, location of the construction in the plan, the materials used and the prefabricated elements), and exploitation (traffic intensity, loads, etc.).

The deformation joint cover devices themselves are a foreign element in the road pavement on the bridge, and therefore it is necessary to correctly calculate the displacements caused by the temperature difference and the directions of the load actions (horizontal, vertical, and angular), as well as the other factors that will influence and to choose the most suitable device capable of absorbing all the factors listed above - to permit interconnection of those devices with the other elements of the construction.

\section{REFERENCES:}

1. SNiP 23-01-99. Construction Climatology. M. GUP CPP, 2000.

2. Improving the design of bridges/V. I. Shesterikov. - M.: Informavtodor, 2002. - Volume 12. p. 25-55.

3. Movement calculations of the mating ends of the span structures of bridge structures and selection of modern designs of expansion joints for use. Guidelines for the implementation of laboratory work. Saratov $2004-33$ p.

4. SNiP 2.05.03-84*. Bridges and Culverts. USSR, 1991

5. Günter Ramberger. Structural bearings and expansion joints for bridges. Structural Engineering Documents 6. Zurich, Switzerland, IABSE, 2002. - p. 51-89.

6. Physical quantities: Directory / A. P. Babichev, N. A. Babushkina, A. M. Bratkovskii, and others: Energoatomizdat, 1991.

7. Belyaev N. M. Strength of materials. -M.: Science, 1976.

8. Study of the stress-strain state of curvilinear spans in the plan and profile and improvement of their manufacturing technology. Chapter 4: Development of initial requirements and programs OKR to create highly reliable expansion joints of bridge structures with large and small movements. CNIIS. M., 1998. 